\title{
Matching Slot Role in Bandwidth Enhancement of SIW Cavity-Backed Slot Antenna
}

\author{
Mohammad Vatankhah Varnoosfaderani, Junwei Lu Senior Member, IEEE, and Boyuan Zhu Member, IEEE \\ School of Engineering, Griffith University, QLD 4111, Australia \\ mohammad.vatankhahvarnoosfaderani@griffithuni.edu.au
}

\begin{abstract}
A substrate-integrated waveguide (SIW) cavitybacked antenna with two slots on top of the cavity has been proposed in this paper. The bowtie slot is the main radiator of the cavity and a rectangular slot close to the feed point has been etched (to add extra resonance to enhance the bandwidth of the antenna in the desired frequency range). Tuning the rectangular slot improved the fractional bandwidth to $8 \%$. A $7.9 \mathrm{dBi}$ peak gain and a steady beam over the entire bandwidth are achieved. The FTBR (front to back ratio) of the antenna is more than 20 dBi. A standard PCB producing process has been used in order to manufacture the proposed antenna on a single layer substrate.

Index Terms - bowtie, slot antenna, cavity-backed, matching slot, substrate-integrated waveguide (SIW).
\end{abstract}

\section{INTRODUCTION}

SIW technology was developed as an alternative to bulky metallic waveguides. Using this technique, a cavity can be implemented in thin planar substrates. This caused a huge size reduction in microwave circuits, which needed a bulky metallic waveguide. Although these waveguides suffer more loss compared with conventional metallic waveguides, they have an acceptable level of performance for a wide range of antenna applications [1-5] and miniaturized filters [6]. This technique enables fabrication of planar integrated rectangular and cylindrical cavities [1-7]. SIW cavity-backed antennas possess the good features of conventional cavity-backed antennas without their drawbacks like bulky cavity resonators and costly fabrication of metallic waveguides.

One of the most important disadvantages of SIW antennas is their coplanar waveguide (CPW) feed lines, which they are commonly fed by. The CPW feed may cause unwanted radiation, which can be easily neglected, but this unwanted radiation will increase cross polarization in more complex structures. As demonstrated in [2], the CPW feeding structure has somehow increased the unwanted feed radiation; however, the length of the CPW feed plays an important role in the determination of its quality factor [3]. Hence, it is not acceptable to shorten the length of the feed line to decrease the unwanted radiation. In [1], feeding and radiating slots are placed on opposite planes to prevent unwanted radiation from adding up to the main lobe. This can degrade the front to back ratio of the antenna because it increases the radiation feed in the reverse direction which is not desirable.

Other drawbacks of SIW cavity-backed antennas include their narrow bandwidth for their low-profile configuration and unique responding mechanism, and their high Q-factor [1]. Increasing substrate thickness is conventionally suggested to increase bandwidth of cavity-backed antennas. In [5], an inductive diaphragm using via arrays with diameters of 0.3 $\mathrm{mm}$ is inserted in the SIW feed. This leads to a more accurate fabrication but it is not cost-effective. In [10], excitation of hybrid modes is used to enhance the bandwidth of the antenna using a long slot.

In this paper, a SIW cavity-backed bowtie slot antenna is presented, as shown in Fig. 1. An extra resonant slot is implemented next to the feed point and in the area where electric fields are strong enough to excite the slot to enhance the bandwidth in the desired frequency range. The slot can be easily fabricated and is not sensitive to fabrication errors like the antenna presented in [9].

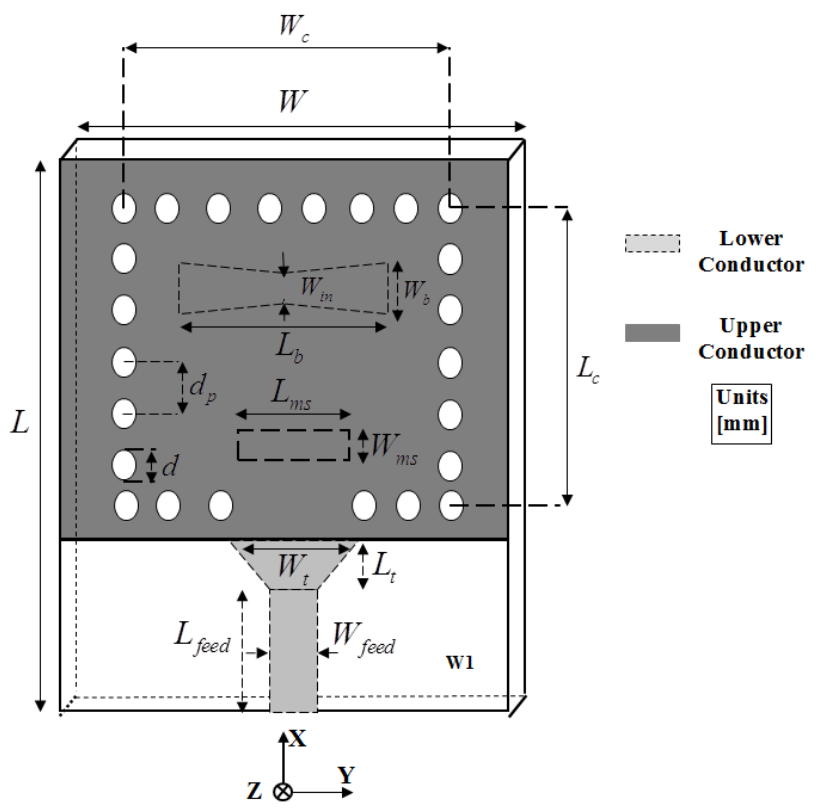

Fig. 1. Geometrical configuration of the proposed antenna

\section{ANTENNA DESIGN}

Based on the dimension of the cavity $\mathrm{TE}_{210}$ mode is excited in the cavity without any slots (Fig. 2). As mentioned in [10], different hybrid modes can be excited by etching a slot on the upper or lower ground plane of the cavity. Size and position of the slot determines if a single mode or combination of hybrid modes would be excited [10] and it can enhance the overall bandwidth of the antenna compared to an antenna with a simple $\lambda / 2$ slot in the middle of the cavity [1]. 




Fig. 2. Electric field profiles of the cavity at $10 \mathrm{GHz}$.

A high-frequency structure simulator (HFSS) is used in order to simulate and optimize the slot size and position. SIW antennas can be modelled by conventional rectangular waveguide structures [8]. By this method, via arrays may be modelled by a finite conductivity rectangular wall in the simulation environment. While this is not a precise method, it can be used in the early stages of simulation in order to understand the overall achieved results. Using walls instead of via arrays can reduce the duration of the simulation. This method can become quite useful in the initial steps of simulation and optimization of more complex structures like multi-layer low temperature co-fired ceramic (LTCC) filters and antennas. In order to receive accurate simulation results of a structure, a final simulation step is needed where rectangular walls are again turned back to via arrays.

In the first design step, an equivalent width of the SIW cavity is chosen from an accurate empirical equation as mentioned in [8]:

$$
w_{\text {eff }}=w-1.08 . \frac{d^{2}}{s}+0.1 \frac{d^{2}}{w}
$$

An antenna with the single bowtie on the upper ground plane is called Ant.1 and is used to show the effect of matching slot on bandwidth improvement. Figure. 3 presents prototypes for both Ant.1 and Ant.2. Return loss of Ant. 1 and Ant. 2 are depicted in Fig. 4 and it can be obviously seen that Ant.1 has a low Q-factor resonance with fractional bandwidth of $4 \%$ at $10.05 \mathrm{GHz}$. Adding the rectangular slot, another resonance appears and improves the fractional bandwidth up to $8 \%$.



Fig. 3. Prototypes of Ant.1 and Ant.2.

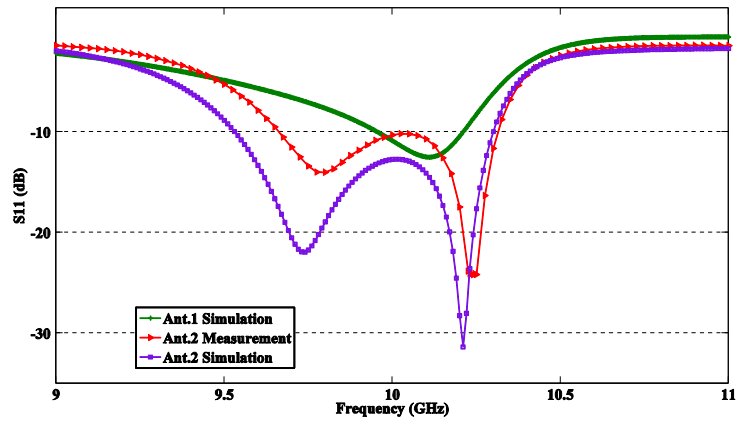

Fig. 4. Measured and simulated S11 of the proposed antennas.

The main advantage of SIW structures, compared to conventional cavity-backed antennas, is that their low profile can be fabricated on a single substrate. The rectangular cavity resonator of an SIW antenna is formed by four walls made of via arrays (Fig. 1).

In order to satisfy the equivalency between the SIW and the conventional metallic cavity, the following conditions have been considered in the design process: $d / d_{p} \geq 0.5$ and $d / \lambda_{0} \leq 0.1$ ( $\lambda_{0}$ is the free space wavelength). Under these circumstances, via diameter and distance between vias are fixed to: $\mathrm{d}=1.5 \mathrm{~mm}$ and $d_{p}=1.9 \mathrm{~mm}$ for the proposed application. In the final design, two slots are etched on the top face of the cavity: a bowtie slot (etched in the area where electric fields are highest in value) and a rectangular slot (etched close to another peak of electric fields in the cavity). These slots add another resonance and act as matching elements as well by having an effect on the feed point of the cavity. For the purpose of measurement, a $50 \Omega$ microstrip line with a tapered transition is used to excite the cavity [11].

In order to enhance the bandwidth of Ant. 1, the matching slot technique is employed. A rectangular matching slot was etched close to the feed point. The antenna with both slots is called Ant. 2 and its final optimized geometrical parameters are presented in Table 1.

TABLE 1

GEOMETRICAL PARAMETERS OF THE PROPOSED ANTENNA

\begin{tabular}{c|c|c|c}
\hline \hline PARAMETER & value $(\mathrm{mm})$ & PARAMETER & value $(\mathrm{mm})$ \\
$\mathrm{h}$ & 0.7874 & $\mathrm{~W}_{\mathrm{ms}}$ & 0.6 \\
$\mathrm{~W}_{\mathrm{C}}$ & 26.7 & $\mathrm{~W}_{\mathrm{t}}$ & 9 \\
$\mathrm{~L}_{\mathrm{C}}$ & 22.65 & $\mathrm{~L}_{\mathrm{t}}$ & 5 \\
$\mathrm{~d}_{\mathrm{P}}$ & 1.9 & $\mathrm{~W}_{\text {feed }}$ & 2.4 \\
$\mathrm{~d}$ & 1.5 & $\mathrm{~L}_{\text {feed }}$ & 10 \\
$\mathrm{~L}_{\mathrm{b}}$ & 22 & $\mathrm{~W}$ & 42 \\
$\mathrm{~W}_{\mathrm{b}}$ & 4.45 & $\mathrm{~L}$ & 40 \\
$\mathrm{~W}_{\mathrm{in}}$ & 0.4 & $\mathrm{~L}_{\mathrm{ms}}$ & 9 \\
\hline
\end{tabular}

By adding the matching slot, currents flowing around the rectangular slot interfere with currents flowing on the top face of the cavity. It acts as a sink for the residual power inside the cavity. As a consequence, two high Q-factor resonances would occur at $9.73 \mathrm{GHz}$ and $10.21 \mathrm{GHz}$. The electric field profiles of the proposed antenna at these frequencies are presented in Fig.5 and the dominant excited mode in the cavity after 
etching the slots is still $\mathrm{TE}_{210}$.

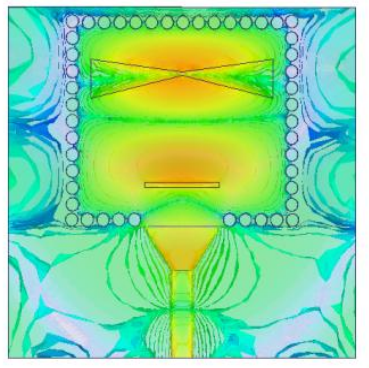

(a)

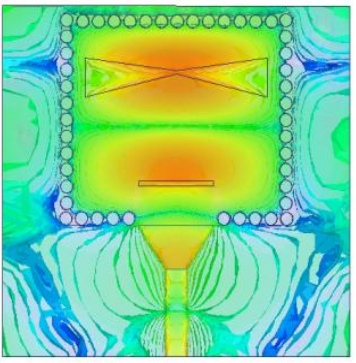

(b)
Fig. 5. Electric field profiles of the antenna, (a) at $9.73 \mathrm{GHz}$ and (b) at 10.21 $\mathrm{GHz}$

Figure 4 makes a comparison between measured and simulated return loss of Ant. 2 as well and a parametric study for the rectangular slot is presented in Fig. 6 (to prove that the rectangular slot participates in changing the lower resonance at $9.73 \mathrm{GHz}$ and improves the bandwidth by having an effect on the feeding mechanism). It is evident that rectangular slot changes lower the resonance frequency of the antenna and do not change the higher frequency resonance. For different dimensions of the rectangular slot, Q-factors of two resonances change and thus the bandwidth of the proposed antenna increases. Etching the matching slots increases the bandwidth from $4 \%$ to $8 \%$, while there are two high Q-factor resonances about $500 \mathrm{MHz}$ and $300 \mathrm{MHz}$ apart at $9.73 \mathrm{GHz}$ and $10.21 \mathrm{GHz}$ respectively.

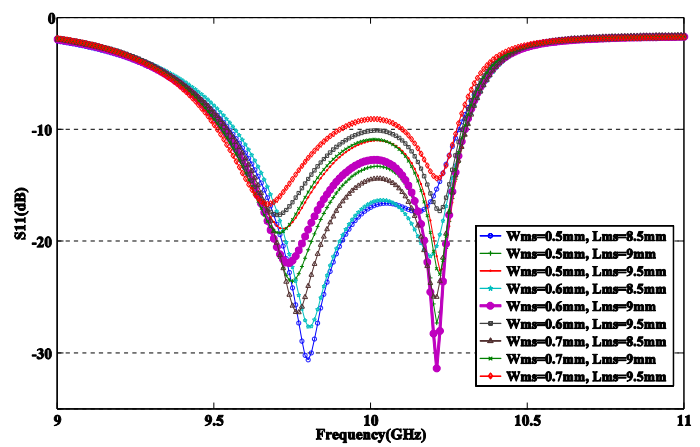

Fig. 6. Simulated S11 of the Ant. 2 versus matching slot dimensions.

\section{ANTENNA MEASUREMENT}

A prototype of the proposed antenna shown in Fig. 1 was fabricated on a RT/Duriod 5880 substrate with thickness of 31 mil, relative permittivity of 2.2 , and loss tangent of 0.0009 . The overall dimensions of the antenna are $42 \mathrm{~mm} \times 40 \mathrm{~mm} \times$ $31 \mathrm{mil}$ and the cavity size is about $26.7 \mathrm{~mm} \times 22.65 \mathrm{~mm}$. The antenna was tested in an anechoic chamber using an Agilent 3115 horn antenna as the reference antenna. Return loss of the antenna was measured as well using a Rohde \& Schwarz (ZVA 24) VNA. A picture of a prototype of Ant. 2 under test is presented in Fig. 7.

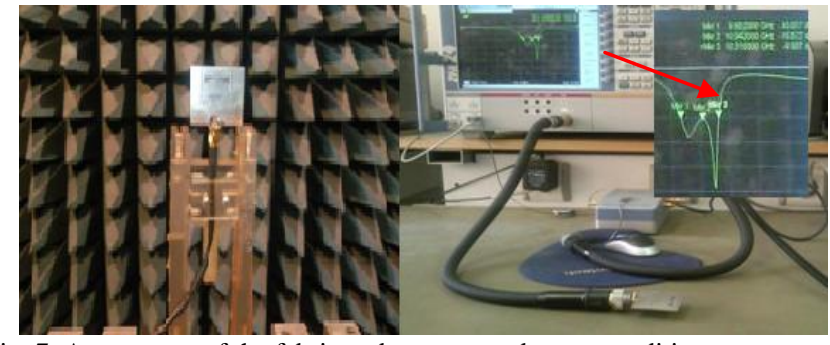

Fig. 7. A prototype of the fabricated antenna under test conditions.

The proposed antenna has a fractional bandwidth of $8 \%$ from $9.53 \mathrm{GHz}$ to $10.3 \mathrm{GHz}$. As can be seen, there are two wide resonances in $9.73 \mathrm{GHz}$ and $10.21 \mathrm{GHz}$. The center frequency is $9.915 \mathrm{GHz}$. Effects of the PCB procedure and finite conductivity of conductor films affects the antenna performance. This, in addition with the finite sized ground plane, is the main reason why high Q-factor resonances are not observed in the measurement results. Conventionally, to overcome the problem of poor ground plane effects, a thick conductor plate may be stuck to the ground plane to improve ground plane performance. For a SIW antenna, which is integrated with follow-up planar circuits, the ground plane will be naturally enlarged and often there is no need for any additional metal sticking.

Fig. 8 shows simulated gain and radiation efficiency. Peak gain is about $7.9 \mathrm{dBi}$ and it occurs at $10.3 \mathrm{GHz}$. As can be seen from this figure, the proposed antenna has relatively high and steady gain over the bandwidth. Measured radiation patterns at 9.9, 10.2 and $10.6 \mathrm{GHz}$ are presented in Fig. 9. Cross polarization is always kept below $-16 \mathrm{dBi}$ and can be neglected. These radiation patterns show that over the entire operational bandwidth, cross polarization in the E-plane ( $\mathrm{x}-\mathrm{Z}$ plane) is far below cross polarization in the H-plane (y-z plane). As the unwanted feed radiation is greatly suppressed, front to back ratio (FTBR) is always better than $20 \mathrm{~dB}$ over the bandwidth. Half power beam widths at different frequencies are presented in Table 2. It is noticeable that the proposed antenna has a wide and steady beam over the operating bandwidth.

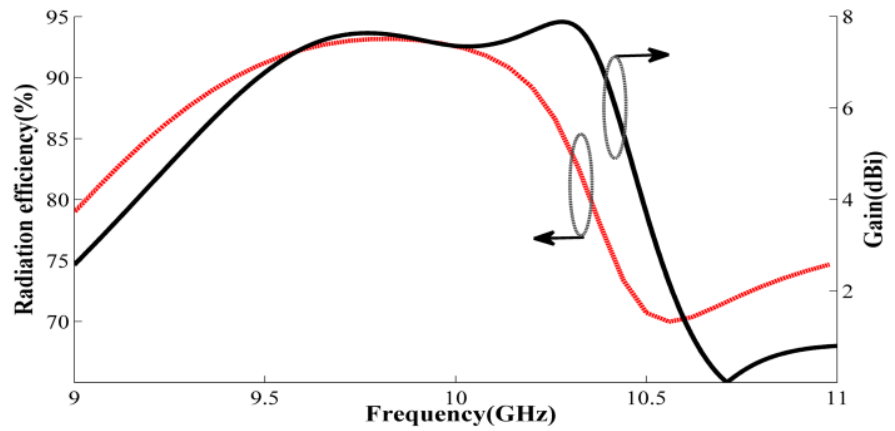

Fig. 8. Simulated gain and efficiency of Ant. 2. 


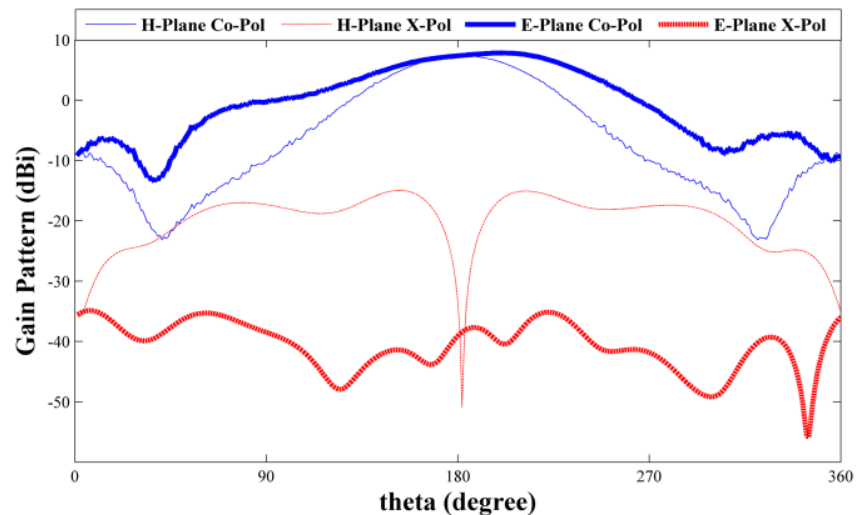

(a)

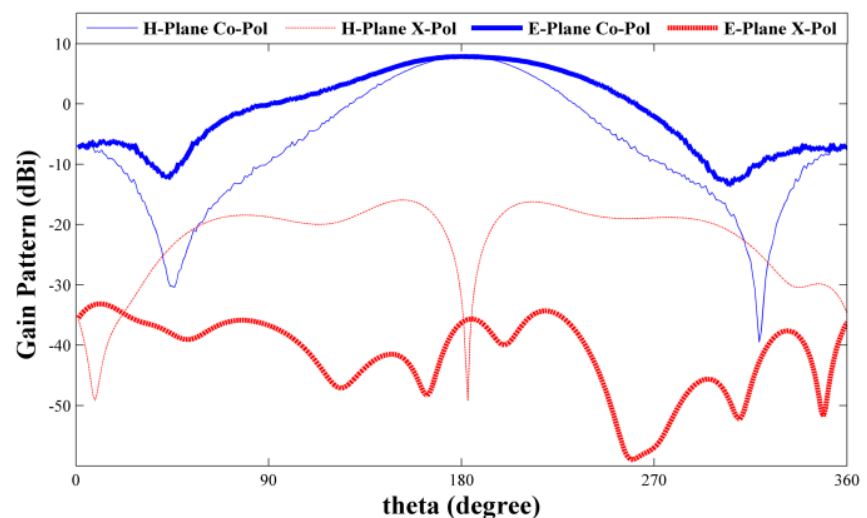

(b)

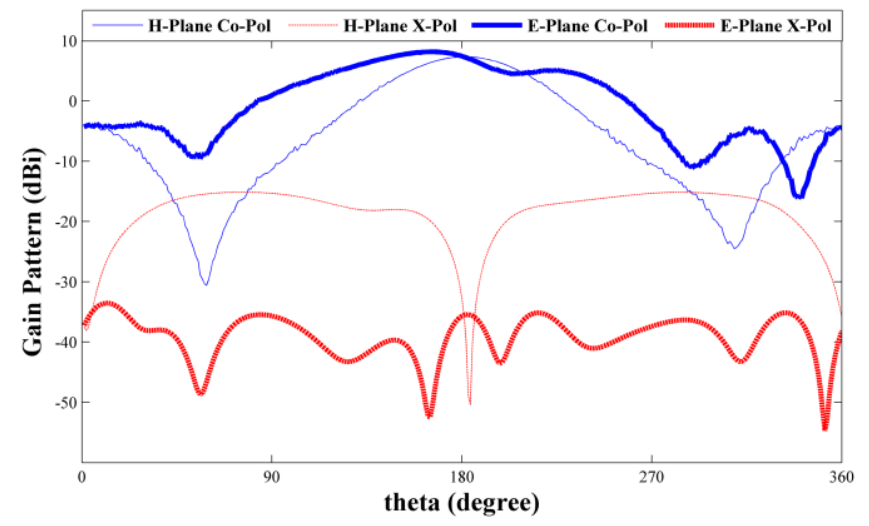

(c)

Fig. 9. Measured radiation patterns for E-plane and H-plane at (a) $9.9 \mathrm{GHz}$, (b) $10.2 \mathrm{GHz}$ and (c) $10.6 \mathrm{GHz}$.

TABLE 2

HALF POWER BEAMWIDTHS

\begin{tabular}{c|c|c}
\hline & H-plane & E-plane \\
\hline $9.9 \mathrm{GHz}$ & $62^{\circ}$ & $93^{\circ}$ \\
\hline $10.2 \mathrm{GHz}$ & $62^{\circ}$ & $93^{\circ}$ \\
\hline $10.6 \mathrm{GHz}$ & $72^{\circ}$ & $60^{\circ}$ \\
\hline
\end{tabular}

\section{CONCLUSION}

A SIW cavity-backed bowtie slot antenna with two slots on the upper ground plane is presented. Both slots are resonant slots and are placed close to the area in which electric fields are highest in value inside the cavity. A simple rectangular slot is acting as a matching slot as well because it is placed close to the feeding point of the cavity and can affect the energy coupling into the cavity. It has been demonstrated that bandwidth enhancement can be achieved by adding the rectangular slot close to the feed point and area in which electric currents are at a maximum. The antenna has relatively high gain $(7-8 \mathrm{dBi})$, which does not change significantly over the whole frequency range of the antenna. The proposed antenna has a wide beam with low cross polarization and satisfies our presumption of high radiation efficiency.

\section{REFERENCES}

[1] Guo Qing Luo, Zhi Fang Hu, Lin Xi Dong, and Ling Ling Sun, "Planar Slot Antenna Backed by Substrate Integrated Waveguide Cavity, "IEEE Antennas Wireless Propag. Lett, vol. 7, pp. 236-239, 2008.

[2] Guo Qing Luo, Zhi Fang Hu, Yaping Liang, Li Yang Yu, and Ling Ling Sun, "Development of Low Profile Cavity Backed Crossed Slot Antennas for Planar Integration" IEEE Trans. Antennas Propag., vol. 57, no. 10, pp. 2972-2979, Oct. 2009.

[3] Juan Carlos Bohórquez, Humberto Adriel Forero Pedraza, Iván Camilo Herrera Pinzón,Jorge Avella Castiblanco, Néstor Peña, and Hector Fabian Guarnizo,"Planar Substrate Integrated Waveguide CavityBacked Antenna, "IEEE Antennas Wireless Propag. Lett, vol. 8, pp. 1139-1142, 2009.

[4] D. Kim, J.W Lee, C.S. Cho and T.K. Lee, "X-band Circular Ring-slot Antenna Embedded in Single-layered SIW for Circular Polarization," Electron. Lett., vol. 45, no. 13, pp. 668-669, Jun. 2009.

[5] Dong-Yeon Kim, Jae W Lee, Taek K. Lee, ChoonSik Cho, "Design of SIW Cavity-Backed Circular-polarized Antennas Using Two Different Feeding Transitions, "IEEE Trans. Antennas Propag., vol. 59, no. 4, pp. 1398-1403, Apr. 2011.

[6] Hung-Yi Chien, Tze-Min Shen, Ting-Yi Huang,Wei-Hsin Wang, and Ruey-Beei Wu, "Miniaturized Bandpass Filters with Double-folded Substrate Integrated Waveguide Resonators in LTCC, "IEEE Trans. Microw. Theory Tech., vol. 57, no.7, pp. 1774-1782, Jul. 2009.

[7] Lorena I. Basilio, Richard L. Chen, Jeffery T. Williams, and David R. Jackson, "A New Planar Dual-band GPS Antenna Designed for Reduced Susceptibility to Low-angle Multipath, "IEEE Trans. Antennas Propag., vol. 55, no. 8, pp. 2358-2366, Aug. 2007.

[8] Feng $\mathrm{Xu}$, and $\mathrm{Ke} \mathrm{Wu}$, "Guided-wave and Leakage Characteristics of Substrate Integrated Waveguide, "IEEE Trans. Microw. Theory Tech., vol. 53, no.1, pp. 66-73, Jan. 2005.

[9] T. Hirano, J. Hirokawa, and M. Ando, "Waveguide Matching Crossedslot", Proc. Inst. Elect. Eng. Microwaves, Antennas Propagation, vol. 150, no. 3, pp. 143-146, Jun. 2003.

[10] Guo Qing Luo, Zhi Fang Hu, Wen Jun Li, Xiao Hong Zhang, Ling Ling Sun, and Jian Feng Zheng "Bandwidth-Enhanced Low-Profile CavityBacked Slot Antenna by Using Hybrid SIW Cavity Modes" IEEE Trans. Antennas Propag., Vol. 60, No. 4, April 2012.

[11] M Vatankhah, M Azarmanesh, AP Saghati, "A novel broadband CBCPW to microstrip transition for concept of implementation in RFcircuits" Journal of Electromagnetic Waves and Applications vol.25 Issue-13, pp.1817-1827, Jan 2011. 